\title{
GaSb and GaSb/AISb superlattice buffer layers for high quality photodiodes grown on commercial GaAs and Si substrates
}

\author{
M. Gutiérrez ${ }^{*}$, F. Lloret ${ }^{1}$, P. Jurczak ${ }^{2}$, J. Wu², H. Y. Liu ${ }^{2}$ and D. Araújo ${ }^{1}$ \\ ${ }^{1}$ Department of Material Science and Metallurgic Engineering, University of Cadiz, Puerto Real 11510, Spain \\ ${ }^{2}$ Department of Electronic and Electrical Engineering, University College London, London WC1E 7JE, UK \\ *marina.gutierrez@uca.es
}

The objective of this work is the integration of $\mathrm{InGaAs} / \mathrm{GaSb} / \mathrm{GaAs}$ heterostructures, with high indium content, on GaAs and Si commercial wafers. The design of an interfacial misfit dislocation array, either on GaAs or Si substrates, allowed to grow strain-free devices. The growth of purposely designed superlattices with their active region free of extended defects on both GaAs and Si substrates is demonstrated. Transmission electron microscopy technique is used for the structural characterization and plastic relaxation study. While in the first case, on GaAs substrates, the presence of dopants was demonstrated to reduce several times the threading dislocation density through a strain hardening mechanism avoiding dislocation interactions, in the second case, on $\mathrm{Si}$ substrates, similar reduction of dislocation interactions is obtained using an $\mathrm{AlSb} / \mathrm{GaSb}$ superlattice. The latter is shown to redistribute spatially the interfacial misfit dislocation array to reduce dislocation interactions.

Keywords: TEM, IMF, Si, photodiode, GaAs

\section{Introduction}

The direct growth of III-V semiconductors on silicon is a great technical challenge to get low cost devices. Nonetheless, it is well known that the large material dissimilarity strongly difficult this integration of III-V heterostructures on silicon. In particular, the extended defects, produced by the large difference in the lattice constants, propagate into the heterostructure and affect the active region of the devices making them unusable.

Concerning GaAs substrates, similar problems occur if high lattice mismatched layers want to be grown. To relieve the large lattice mismatch between the GaSb-based devices structure and the GaAs ( 7.8\%) substrate, the material generates itself a highly periodic array of misfit dislocations, called interfacial misfit (IMF) dislocation array ${ }^{1,2,3,4}$ at the substrate/heterostructure interface. Although thermodynamically unexpected, $60^{\circ}$ misfit dislocations (MDs) are normally observed in addition to the stable edge dislocations. Several models have been reported ${ }^{5,6,7}$ to explain their presence, nonetheless, their interaction with the $90^{\circ} \mathrm{MDs}$ is responsible of the threading dislocation (TDs) generation. In this paper different heterostructure architectures are proposed to get GaSb-based devices structures free of threading dislocation on commercial substrates, i.e. either Si or GaAs substrates. Firstly, the use of a solid solution strengthening 
mechanism, by the addition of Be dopants, is demonstrated to reduce the TDs density in GaSb-based devices grown on GaAs. Secondly, the use of a AlSb/GaSb superlattice with an AlSb nucleation layer, demonstrate to achieve the same purpose but here on Si substrate: lower the misfit dislocation interactions to reduce the threading dislocation generation. The use of such a buffer is able to accommodate the significant lattice mismatch between GaSb and Si $(\sim 12 \%)$ allowing to get an GaSb-based heterostructure on a commercial silicon wafer with a low density of TDs.

\section{Experimental}

Undoped GaSb and Be-doped GaSb (GaSb:Be) layers, samples A and B, were grown on GaAs (001) by molecular beam epitaxy. In both n-type epi-ready GaAs (001) wafers were used as substrates. The GaAs native oxide was desorbed at $600{ }^{\circ} \mathrm{C}$ for $10 \mathrm{~min}$. After oxide desorption, a $200 \mathrm{~nm}$ thick GaAs layer was grown at $590{ }^{\circ} \mathrm{C}$. After the growth of GaAs layer, As flux was immediately stopped. After about $20 \mathrm{sec}$, Sb flux was immediately supplied and the RHEED pattern has changed from $2 \times 4$ to $2 \times 8$. The temperatures were calibrated by a pyrometer. The growth temperature was then reduced from 590 to $510^{\circ} \mathrm{C}$ to grow a 150 -nm-thick undoped GaSb layer, for sample $\mathrm{A}$, and $\mathrm{Be}$ doped to $5 \times 10^{18} \mathrm{~cm}^{3} \mathrm{GaSb}$ layer, for sample B. The doping concentration is calibrated by previous SIMS measurement. For the growth of sample $\mathrm{C}, \mathrm{Si}(001)$ substrate $4^{\circ}$ offcut towards the $<011>$ direction to suppress the formation of antiphase domains was used. Prior to epitaxy growth, the Si substrate was heated in ultra-high vacuum at $900^{\circ} \mathrm{C}$ for $30 \mathrm{~min}$ to fully remove surface native oxide. After deoxidation, the substrate was cooled down to $400^{\circ} \mathrm{C}$ and a $10 \mathrm{~nm} \mathrm{AlSb}$ nucleation layer was deposited by the migration enhanced epitaxy. Sequentially, the growth temperature was raised to about $500^{\circ} \mathrm{C}$ and a buffer was grown at the same substrate temperature. The buffer is made of $100 \mathrm{~nm} \mathrm{AlSb} / \mathrm{GaSb}$ superlattice, $500 \mathrm{~nm} \mathrm{GaSb}$, and a second $100 \mathrm{~nm} \mathrm{AlSb/GaSb}$ superlattice. The superlattice, of totally $100 \mathrm{~nm}$ in thickness was made from alternating GaSb and AlSb layers. The growth of the InAs/GaSb superlattice was then carried out at a lower substrate temperature $\left(400^{\circ} \mathrm{C}\right)$. Each period consisted of $10 \mathrm{ML} \mathrm{GaSb}$ and $10 \mathrm{ML}$ InAs. Since the lattice constant of InAs is $0.75 \%$ smaller than that of GaSb and a thin InSb layer within 1 monolayer was inserted between the InAs and GaSb layers for strain balance (see Fig.1).

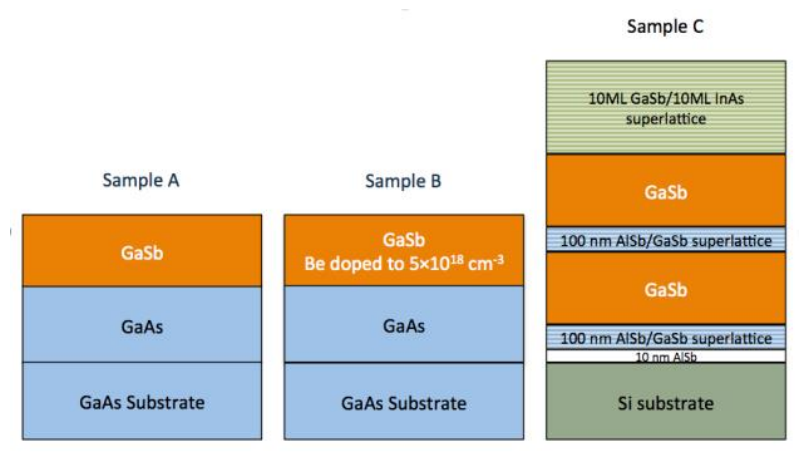

Fig.1. Schema of the studied samples. The effect of doping on the dislocation distribution in GaSb layers grown on semi-coherent GaAs substrate was analysed using samples A and B. Sample C showed that the joint use of a semi-coherent substrate and a buffer layer conforms a strategy to get GaSb based heterostructures on commercial Si wafers. 
Samples were studied using cross-section transmission electron microscopy (TEM). The TEM specimens were prepared using mechanical polishing techniques, followed by Ar+ ion sputtering at liquid-nitrogen temperature. TEM observations were carried out on a Philips CM 200 and a JEOL 2100 EX TEM operated at $200 \mathrm{KeV}$.

\section{Results and discussion}

As expected, high-resolution transmission electron microscopy (HREM) micrographs of sample A evidenced an IMF dislocation array. This highly periodic array of perfect MDs was located in the GaSb/GaAs interface plane and dislocations followed the $\langle 110\rangle$ directions. The distance among these dislocations was found to be $5.7 \mathrm{~nm}$. The result was a semi-coherent interface where two extra $\{111\}$ planes were symmetrically located at the dislocation core. However, these were not the only dislocations found at the interface, $60^{\circ}$ MDs were also observed and, as consequence of the interaction between both types, TDs were generated ${ }^{8,9}$. Qian et al. ${ }^{10}$ proposed that $90^{\circ} \mathrm{MDs}$ at the $\mathrm{GaSb} / \mathrm{GaAs}$ interface are generated at the leading edges during the Volmer-Weber growth model. At a certain moment of the GaSb islands growth, they reach a critical size from which $60^{\circ}$ MDs are generated. Then, the latter glide down to the $\mathrm{GaSb} / \mathrm{GaAs}$ interface and interact with the $90^{\circ} \mathrm{MDs}$ to generate $\mathrm{TDs}^{11}$.

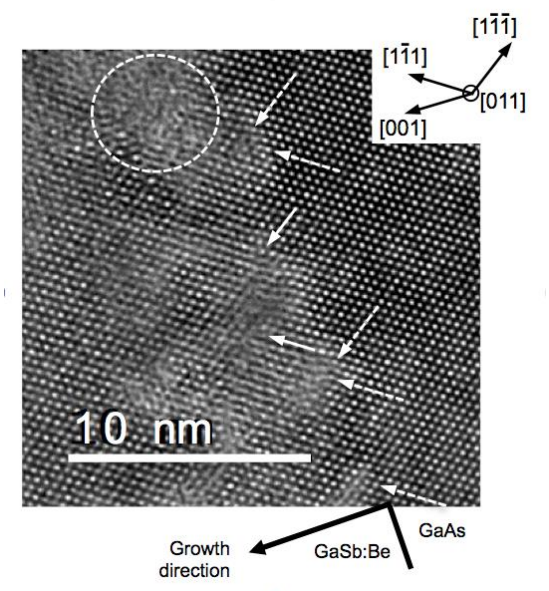

Fig.2. HREM micrograph of sample B where two kinds of misfit dislocations can be observed. $90^{\circ} \mathrm{MDs}$, located in the GaSb:Be/GaAs interface (pointed out with two dashed arrows and $60^{\circ} \mathrm{MDs}$ ) and out of the interface plane (pointed out with continuous arrows). The dashed circle marks an area where it is not possible to see the extra planes. The crystallographic directions as the $\mathrm{GaSb} / \mathrm{GaAs}$ interface have been indicated.

The HREM studies revealed that in sample B, there are two types of areas at the interface. On one hand, there were areas with $90^{\circ} \mathrm{MDs}$ sited at the GaSb:Be/GaAs interface plane. On the other hand, there were areas where, besides the $90^{\circ} \mathrm{MDs}, 60^{\circ} \mathrm{MDs}$ were observed but in this case they were not located at the interface plane. Fig. 2 show one of them, $90^{\circ} \mathrm{MD}$ dislocations (dashed arrows), $60^{\circ} \mathrm{MD}$ dislocations (continuous arrows) and areas where it is impossible to draw the dislocations with precision (dashed circles) are revealed. Thus, in sample B the interface has two behaviours, on one hand there are zones, approximately $200 \mathrm{~nm}$ length, where only $90^{\circ} \mathrm{MDs}$ are observed, on the other hand there are zones, approximately $30 \mathrm{~nm}$ length, where $60^{\circ} \mathrm{MDs}$ are pinned and located outside the GaSb/GaAs interface plane. Selected area electron diffraction (SAED) pattern recorded at the interface showed no coincidence of GaAs and GaSb spots, which allowed to calculate a relaxation rate of the GaSb layer around $98 \%$. Moreover, the spot corresponding to 
one of the $<111>$ directions was tilted $2^{\circ}$. Kang et al. ${ }^{12}$ have explained this observation as an asymmetric distribution of $60^{\circ} \mathrm{MDs}$ at the interface. He argued that this configuration is energetically more favourable than a symmetric one which is in accordance with our results.

The comparison of cross-sectional 220 bright field TEM micrographs of samples A and B with the same sample thickness, magnification and angle, confirm that the TD density in sample B was three times less respect to sample A. So, a solid solution hardening mechanism has been proved to be efficient in this type of samples to get low TD density in the active region of GaSb-based devices grown on GaAs.

With the ultimate goal of obtaining III-V devices on cheap commercial substrates, sample C was grown. In this sample, an AlSb layer was grown on top of the silicon substrate to create a virtual substrate, i.e. to generate an IMF dislocation array that relieves most of the strain energy between the GaSb layer and the silicon, in high similarity with the IMF array formed at the GaSb/GaAs interface in samples A and B (see Fig.3.a). The SAED pattern of Fig.3.b was taken at the interface in the $\langle 110\rangle$ direction showing a double pattern. Based on the silicon lattice parameter, an almost fully $(\sim 95 \%)$ relaxed heterostructures was confirmed. It is remarkable that, in this SAED pattern, there is not rotation of the heterostructure respect to the substrate, as observed in sample B, which moreover contrast with previous results of several authors ${ }^{13}$.
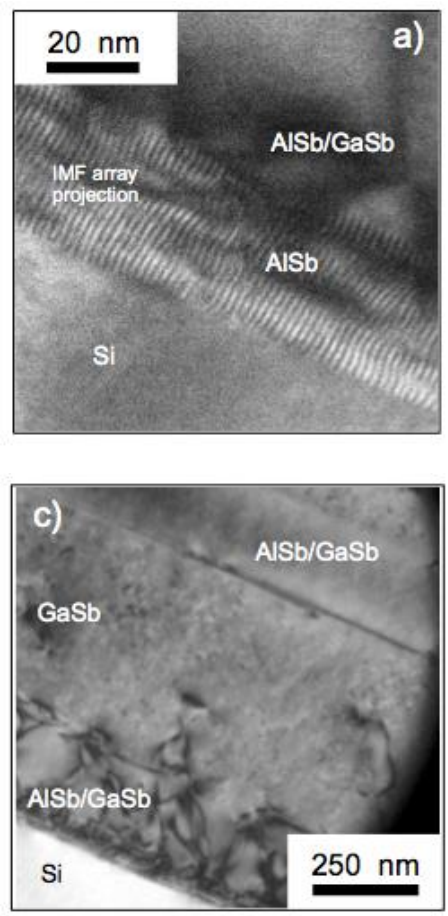

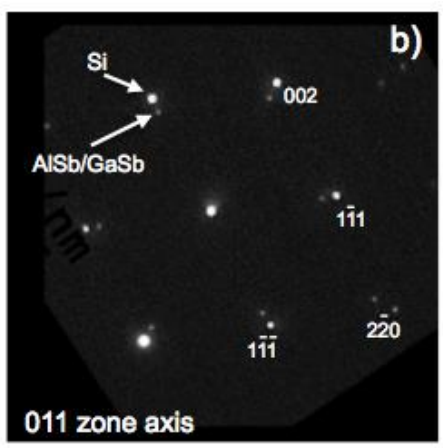

Fig.3. TEM observations of sample C. (a) Cross sectional 220 bright field TEM micrographs of sample $\mathrm{C}$ where the projection of the IMF dislocation array at the $\mathrm{AlSb} / \mathrm{Si}$ interface is observed. (b) SAED pattern of the micrograph (a). (c) TDs, observed in 220 bright field TEM conditions, confined on the $\mathrm{AlSb} / \mathrm{GaSb}$ buffer layer.

Figure.3.c. is a cross-sectional TEM micrograph taken under 220 bright field conditions of the sample C. It is observed how the glide up, from the AlSb/silicon interface, of a high density of TDs is effectively stopped by the use of a thin $\mathrm{AlSb} / \mathrm{GaSb}$ buffer. A distribution of the MD at different height in the layer is observed which results in a reduction of the dislocation interactions. This results from the AlSb/GaSb superlattice strain modulation which can move dislocation 
segments. In addition, the authors are currently studying the influence of doping with Be in these heterostructures which can also pin the dislocations and reduce even more dislocation interactions.. It is expected that the joint effects of: I. the solid solution hardening, II. the IMF dislocation array technique, and III. a fine buffer layer, allow to establish the MBE growth parameters to get InAs/GaSb type II photodiodes on silicon substrates free of extended defects on the active region.

\section{Conclusions}

GaSb layers have been possible to be grown with a low density of extended defects on commercial GaAs and Si wafers. Interfacial misfit dislocation array has been used to get virtual substrates for the optimal growth of these heterostructures. In the case of GaAs substrates, Be dopants have been demonstrated to reduce the extended defect density when $\mathrm{GaSb}$ is grown on virtual GaAs substrate. The unwanted $60^{\circ}$ misfit dislocations formed at the edge of the islands during the Volmer-Weber growth of GaSb result pinned by Be dopants. This avoids the interaction with the $90^{\circ}$ MDs of the IMF dislocation array and so the TD generation which would degrade the active region on a device. On the other hand, on virtual Si substrates the use of a thin AlSb/GaSb buffer layer has allowed to grow GaSb layer almost free of extended defects. It is expected, in this case, that the use of Be dopants allow getting GaSb-based photodiodes free of defects on the active region.

\section{Acknowledgments}

This work has been supported by Spanish Ministry of Economy and Competitiveness (Ref.: TEC2014-54357-C2-2-R, HiVolt-nano project)

\section{Reference List}

1 S. H. Huang, G. Balakrishnan, A. Khoshakhlagh, A. Jallipalli, L. R. Dawson and D. L. Huffaker, Appl. Phys. Lett. 88, 131911 (2006).

2 W. Zhou, X. Li, S. Xia, J. Yang, W. Tang and K. M. Lau, J. Mater. Sci. Technol. 28, 132 (2012).

3 K. H. Tan, B. W. Jia, W. K. Loke, S. Wicaksono and S. F. Yoon, J. Cryst. Growth 427, 80 (2015).

4 B. C. Juang, R. B. Laghumavarapu, B. J. Foggo, P. J. Simmonds, A. Lin, B. Liang and D. L. Huffaker, Appl. Phys. Lett. 106, 111101 (2015).

5 P. E. Hopkins, J. C. Duda, S. P. Clark, C. P. Hains, T. J. Rotter, L. M. Phinney and G. Balakrishnan, Appl. Phys. Lett. 98, 161913 (2011).

6 Y. Wang, P. Ruterana, S. Kret, S. El Kazzi, L. Desplanque and X. Wallart, Appl. Phys. Lett. 102, 052102 (2013).

7 Y. Wang, P. Ruterana, L. Desplanque, S. El Kazzi and X. Wallart, J. Appl. Phys. 109, 023509 (2011).

8 Q. Dai, M. F. Schubert, M. H. Kim, J. K. Kim, E. F. Schubert, D. D. Koleske, M. H. Crawford, S. R. Lee, A. J. Fischer, G. Thaler and M. A. Banas, Appl. Phys. Lett. 94, 111109 (2009). 
9 D. Cherns, S. J. Henley and F. A. Ponce, Appl. Phys. Lett. 78, 2691 (2001).

10 W. Qian, M. Skowronski, R. Kaspi, M. De Graef and V. P. Dravid, J. Appl. Phys. 81, 7268 (2007).

11 L. Vescan, W. Jager, C. Dieker, K. Schmidt, A. Hartmann and H. Luth, Mater. Res. Soc. Symp. 263 , 23 (1992).

12 J. M. Kang, M. Nouaoura, L. Lassabatere and A. Rocher, J. Cryst. Growth 143, 115 (1994).

13 G. Balakrishanan, S. Huang, L. R. Dawson, Y. C. Xin, P. Collin and D. L. Huffaker, Appl. Phys. Lett. 86, 034105 (2005).

\section{Figure captions}

Fig.1. Schema of the studied samples. The effect of doping on the dislocation distribution in GaSb layers grown on semi-coherent GaAs substrate was analysed using samples A and B. Sample C showed that the joint use of a semicoherent substrate plus a buffer layer conforms a strategy to get GaSb based heterostructures on commercial Si wafers.

Fig.2. HREM micrograph of sample B where two kinds of misfit dislocations can be observed. $90^{\circ}$ MDs, located in the GaSb:Be/GaAs interface (pointed out with two dashed arrows and $60^{\circ} \mathrm{MDs}$ ) and out of the interface plane (pointed out with continuous arrows). The dashed circle marks an area where it is not possible to see the extra planes. The crystallographic directions as the $\mathrm{GaSb} / \mathrm{GaAs}$ interface have been drawn.

Fig.3. TEM observations of sample C. (a) Cross sectional 220 bright field TEM micrographs of sample C where the projection of the IMF dislocation array at the AlSb/Si interface is observed. (b) SAED pattern of the micrograph (a). (c) TDs, observed in 220 bright field TEM conditions, confined on the AlSb/GaSb buffer layer. 